\title{
Distributed Computing in Opportunistic Environments
}

\author{
Mohan Kumar \\ The University of Texas at Arlington, USA
}

\begin{abstract}
Opportunistic networks have evolved from mobile ad hoc networking and the delayed tolerant networking paradigms and have quickly gained popularity in research and industry. In opportunistic networking, when pairs of devices come within each others communication range, opportunistically, short-lived links (or opportunistic links) are created. Opportunistic computing exploits the opportunistic links created by pair-wise contacts, to share information content, resources and services, leading to a wide variety of applications. In this talk we discuss the research challenges and issues in exploiting opportunistic contacts to create a delay tolerant distributed computing environment. Further more, the impact of social computing and networking paradigms for such problems as synchronization and trust will be investigated. Results of ongoing research work on information dissemination, collaboration and trust will be presented. The research work is supported through an NSF grant.
\end{abstract}

